\title{
QUANTIFICAÇÃO DAS FASES CONSTITUINTES DE AGREGADOS RECICLADOS POR ANÁLISE DE IMAGENS AUTOMATIZADA
}

\author{
H. KAHN ${ }^{1}$; C. ULSEN ${ }^{1}$; R. R. FRANÇA ${ }^{2}$; G. HAWLITSCHEK ${ }^{1}$ e R. CONTESSOTTO ${ }^{1}$ \\ Universidade de São Paulo, Escola Politécnica. ${ }^{1}$ Depto de Eng. de Minas e de Petróleo. ${ }^{2}$ Instituto de Geociências. \\ henrique@lct.poli.usp.br; carina@Ict.poli.usp.br
}

Artigo submetido em novembro/2013 e aceito em março/2014

http://dx.doi.org/10.15628/holos.2014.1787

\section{RESUMO}

A demanda atual pela utilização de materiais reciclados na construção deve-se ao aumento crescente do consumo de agregados, à exaustão de jazidas próximas aos grandes centros e à proibição de deposição de resíduos inertes em aterros sanitários. Paralelamente, com o crescimento evidente do setor da construção em muitos países, o gerenciamento dos resíduos de construção e demolição tornou-se uma questão econômica e ambiental prioritária. A principal dificuldade na produção de areia reciclada está relacionada à quantificação da pasta de cimento proveniente de construções anteriores que se mantém aderida à superfície das partículas e aumenta a porosidade e área de superfície específica dos agregados reciclados.
Portanto, a caracterização e quantificação dessa fase é essencial para a aplicação deste produto e uma das ferramentas, ora proposta, é por meio da caracterização microestrutural. O escopo do trabalho envolveu a caracterização tecnológica de agregados miúdos produzidos a partir de resíduos de construção e demolição, por meio de separações de fases (minerais e outras), análises químicas, determinação do teor de pasta de cimento e detalhamento da composição mineralógica de cada produto analisado e das associações das fases por microscopia eletrônica de varredura. As correlações estabelecidas e a coerência dos resultados apresentados demonstram a robustez e confiabilidade do procedimento estabelecido.

PALAVRAS-CHAVE: agregados miúdos reciclados, liberação, análise de imagens.

\section{AUTOMATED IMAGE ANALYSIS ON THE QUANTIFICATION OF RECYCLED AGGREGATES PHASES}

\begin{abstract}
The current demand for the use of recycled materials in construction is due to the increasing consumption of aggregates, the depletion of important deposits close to large centers and the prohibition of disposal in landfills. Besides, the growth of the building sector is evident and the management of construction and demolition waste has become a priority in many countries. The main difficulty in the use of recycled sand is related to the quantification of the cement paste from previous constructions which remains adhered to the surface of the particles and increases the porosity and specific surface area of the recycled aggregates. Therefore, the
\end{abstract}

characterization and quantification of this phase are essential for the application of this product. The scope of this work involved the technological characterization of aggregates produced from construction and demolition waste by phase's separations (minerals and other phases), chemical analysis, quantification of cement paste and detailed mineralogical composition of each product and the phase's association by scanning electron microscopy. The correlations established and consistencies of the results show the robustness and reliability of the established procedure.

KEYWORDS: fine recycled aggregates, liberation, image analysis. 


\section{INTRODUÇÃO}

Atualmente, a principal aplicação para os agregados reciclados a partir de resíduos de construção e demolição, os chamados RCD, é em atividades de pavimentação, uma vez que não exigem propriedades de alta qualidade dos materiais (HENDRIX, 2000). A elevada porosidade dos RCD, oriunda do conteúdo de pasta de cimento remanescente de construções anteriores, é o que origina os maiores problemas nas suas aplicações (BARRA e VAZQUEZ, 1998; DE JUAN e GUTIERREZ, 2009): alta porosidade e absorção de água, elevado índice de abrasão Los Angeles e do ponto de vista mecânico, afeta também a elasticidade, a durabilidade e a resistência à compressão.

Alternativas de processo que sejam capazes de remover a fração porosa dos RCD têm sido alvo de investigações tanto no Brasil como na Europa (ANGULO, 2005; ULSEN, 2006). Contudo, esses estudos focam apenas a fração graúda dos RCD. O processamento da fração areia (agregados miúdos) por técnicas de tratamento de minérios tem sido pouco investigado, embora Hendrix (HENDRIKS, 2004) aponte algumas operações passíveis de serem aplicadas para este produto. Da mesma forma são poucos os trabalhos que investigam os procedimentos de caracterização desta fração, que representa de 40 a 60\% em massa de resíduos brasileiros (ULSEN, HAWLITSCHEK et al., 2010).

Não existem métodos normatizados para determinação do teor de pasta de cimento em agregados reciclados. Os principais procedimentos descritos na literatura envolvem lixiviação por ácido clorídrico e quantificação da soma dos teores de $\mathrm{CaO}$ com a perda ao fogo (ANGULO, ULSEN et al., 2009). No entanto, a presença de agregados calcários na composição do resíduo inviabiliza tal aproximação, uma vez que os carbonatos são também solubilizados por ácido clorídrico, constituídos por $\mathrm{CaO}$ e com elevados valores de perda ao fogo.

Neste trabalho é discutida a separabilidade das fases presentes em agregados miúdos reciclados, por meio de separações sequenciais em líquidos densos e caracterização dos produtos por análises químicas e por análise de imagens por feixe de elétrons.

\section{MATERIAIS E MÉTODOS}

Os estudos foram conduzidos em areias recicladas produzidas a partir da britagem terciária de resíduos de construção e demolição (RCD). O procedimento experimental envolveu as atividades abaixo sumarizadas:

Amostragem: inicialmente foi realizada uma amostragem de RCD britados (britador de impacto) na usina de reciclagem Urbem Tecnologia Ambiental, localizada no município de São Bernardo do Campo, região metropolitana de São Paulo, durante 60 dias, duas vezes por semana, até a obtenção de 4 toneladas de amostra. Uma descrição detalhada na usina é apresentada por Ulsen (ULSEN, 2011).

Britagem secundária: Todo material amostrado foi rebritado em britador de mandíbulas no Laboratório de Tratamento de Minérios e Resíduos Industriais da Escola Politécnica da USP para obtenção do material abaixo de $19 \mathrm{~mm}$.

Britagem terciária: Após a britagem secundária, o material foi transportado para o Centro de Tecnologia de Processos (CTP) da Metso, onde foi alimentado ao britador de impacto vertical 
(VSI modelo Barmac 3000) para a britagem terciária. A operação foi realizada sem cascata, em circuito fechado com peneira de $3,0 \mathrm{~mm}$ e velocidade de rotação de $75 \mathrm{~m} / \mathrm{s}$. O agregado miúdo reciclado $(<3 \mathrm{~mm}$ ) foi transportado para o Laboratório de Caracterização Tecnológica da Escola Politécnica da USP para estudos subsequentes. Detalhes do procedimento de produção do agregado miúdo reciclado são apresentados por Ulsen e colaboradores. (ULSEN, KAHN et al., 2013).

Preparação de amostras para MEV-MLA: O produto da britagem terciária, denominado VSI75 foi homogeneizado e amostrado para obtenção de alíquotas representativas para os estudos de caracterização. Uma alíquota da amostra foi peneirada em peneiras de aberturas nominais de 2,$0 ; 1,2 ; 0,60 ; 0,30$ e $0,15 \mathrm{~mm}$ e, na sequência, cada fração foi objeto se separações sequenciais em líquido denso nas densidades de 2,2; 2,5;2,6 e 2,64 g/cm3 (mistura de bromofórmio e álcool etílico). Ao final foram obtidos cinco produtos de densidade em cinco frações granulométricas, totalizando vinte e cinco produtos. A separação por densidade é particularmente interessante para a separação de partículas de diferente massa específica aparente e, portanto, porosidades distintas (ANGULO, 2005), quanto maior a porosidade, menor a massa específica aparente, e portanto, menor a resistência mecânica da partícula.

Caracterização dos produtos: Determinação da composição química dos produtos por espectrometria de fluorescência de raios $X$ (FRX) em pérolas fundidas (análises quantitativas) em equipamento Axios Pro, PANalytical. Determinação da perda ao fogo a 1.050 C por $1 \mathrm{~h}$. Análise da composição mineralógica por difratometria de raios $X$, método do pó, em equipamento PANalytical com radiação de $\mathrm{Cu} K \alpha$.

Quantificação das fases presentes por MEV-MLA: A composição das fases presentes e as respectivas associações foram avaliadas por meio de análise de imagens automatizada (FANDRICH, GU et al., 2007) em seções polidas. Foi utilizado o software MLA - Mineral Liberation Analyser (FEI) acoplado ao microscópio eletrônico de varredura (MEV), modelo Quanta 600 FEG (FEI), equipado com sistema de microanálise por EDS Esprit-Quantax 4030 (tecnologia SDD, silicon drift detector), marca Bruker.

Inicialmente foram coletadas imagens de elétrons espalhados (BSE) com discriminação das fases de diferentes tons de cinza e posterior coleta de espectros de raios X por EDS para um mapeamento por composição química em uma malha de pontos uniforme e densa, de modo a discriminar fases cujas tonalidades na imagem de BSE são muito semelhantes.

Após a coleta, foi realizada a classificação das partículas. Os espectros de raios $\mathrm{X}$ coletados são sistematicamente comparados com aqueles das fases presentes do banco de dados e os grãos são classificados de acordo com sua equivalência, gerando uma imagem digital da seção onde cada fase individualizada é representada por uma cor. Posteriormente, recursos de análise de imagens disponíveis no software permitem a obtenção de informações sobre as proporções de cada fase e suas respectivas formas de associação e liberação.

O objetivo foi desenvolver um procedimento de caracterização hábil na determinação da composição e das associações das fases presentes nos produtos das separações por líquido denso, bem como avaliar a liberação entre as fases de interesse a pasta de cimento. 


\section{RESULTADOS E DISCUSSÕES}

\subsection{Separabilidade por líquidos densos}

As curvas de separabilidade por densidade, com valores acumulados nos produtos afundados são apresentadas na Figura 1 e a composição química dos produtos (total -3,0+0,15 $\mathrm{mm}$ ) na Tabela 1.

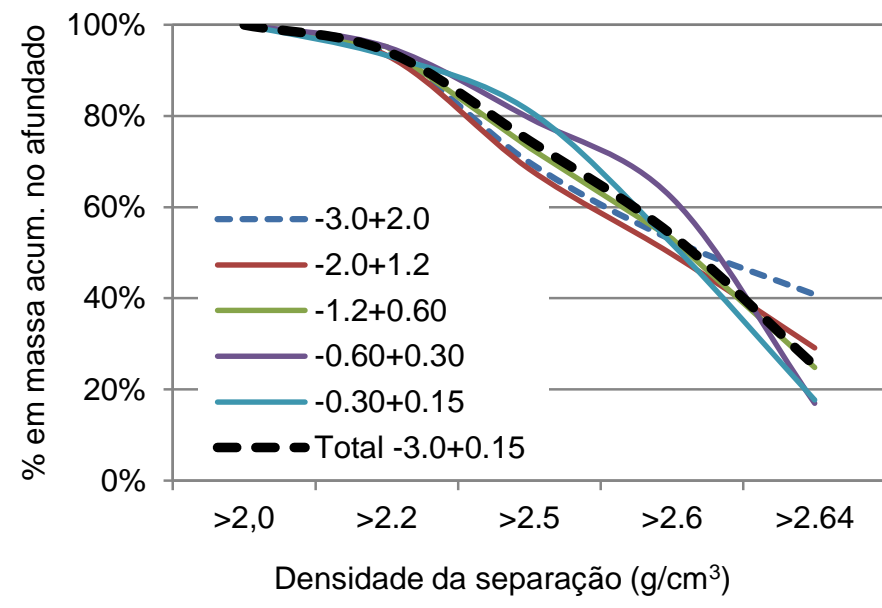

\begin{tabular}{|c|c|c|c|c|c|c|}
\hline \multirow{2}{*}{$\begin{array}{l}\text { Produtos } \\
\left(\mathrm{g} / \mathrm{cm}^{3}\right)\end{array}$} & \multicolumn{6}{|c|}{ Fração (mm); \% em massa } \\
\hline & $+2,0$ & $+1,2$ & $+0,60$ & $+0,30$ & $+0,15$ & Tot \\
\hline & 3 & & & 95.2 & & \\
\hline & 69,8 & 68 & 73,0 & 79,6 & 011 & 6 \\
\hline$d>2,6 c$ & 52,8 & 49 & 53,1 & 62,0 & 51,9 & 53 \\
\hline$d>2,64$ & 40,8 & 29,2 & 24,8 & 16,9 & 17,7 & 25 \\
\hline
\end{tabular}

Figura 1 - Curvas de separabilidade por densidade

Tabela 1 - Composição química dos produtos das separações em líquido denso, total calculado -3,0+0,15 mm

\begin{tabular}{|c|c|c|c|c|c|c|c|c|c|c|c|c|c|c|c|}
\hline \multirow{2}{*}{$\begin{array}{l}\text { Fração } \\
\text { (mm) }\end{array}$} & \multirow{2}{*}{$\begin{array}{c}\text { Massa } \\
\text { (\%) }\end{array}$} & \multicolumn{9}{|c|}{ Teores (\%) } & \multicolumn{5}{|c|}{ Distribuição (\%) } \\
\hline & & $\mathrm{SiO}_{2}$ & $\mathrm{Al}_{2} \mathrm{O}_{3}$ & $\mathrm{Fe}_{2} \mathrm{O}_{3}$ & $\mathrm{Na}_{2} \mathrm{O}$ & $\mathrm{K}_{2} \mathrm{O}$ & MgO & $\mathrm{CaO}$ & PF & $\begin{array}{l}\mathrm{CaO} \\
+\mathrm{PF}\end{array}$ & $\mathrm{SiO}_{2}$ & $\mathrm{Al}_{2} \mathrm{O}_{3}$ & $\mathrm{Fe}_{2} \mathrm{O}_{3}$ & $\begin{array}{l}\mathrm{CaO} \\
+\mathrm{PF}\end{array}$ & $\begin{array}{r}\mathrm{Na}_{2} \mathrm{O} \\
+\mathrm{K}_{2} \mathrm{O}\end{array}$ \\
\hline \multicolumn{16}{|l|}{$-3,0+0,15$} \\
\hline$d<2,2$ & 5,8 & 44,2 & 7,36 & 2,89 & 2,32 & 0,42 & 1,22 & 23,3 & 16,2 & 39,5 & 3,7 & 5,2 & 5,0 & 17,5 & 2,5 \\
\hline $2,2<d<2,5$ & 19,6 & 59,2 & 7,08 & 2,59 & 1,61 & 0,58 & 2,26 & 14,1 & 10,4 & 24,5 & 17,0 & 17,1 & 15,3 & 36,9 & 14,7 \\
\hline $2,5<d<2,6$ & 20,7 & 70,4 & 9,90 & 2,49 & 0,63 & 1,34 & 4,45 & 4,51 & 3,75 & 8,26 & 21,3 & 25,2 & 15,4 & 13,1 & 31,6 \\
\hline $2,6<d>2,64$ & 28,7 & 85,1 & 5,19 & 2,22 & 1,30 & 1,28 & 0,23 & 1,82 & 1,40 & 3,22 & 35,7 & 18,3 & 19,1 & 7,08 & 19,5 \\
\hline$d>2,64$ & 25,2 & 60,3 & 11,0 & 5,97 & 2,37 & 2,41 & 2,14 & 7,87 & 5,27 & 13,1 & 22,2 & 34,2 & 45,2 & 25,4 & 31,7 \\
\hline Total calc. & 100 & 68,4 & 8,14 & 3,34 & 1,19 & 1,39 & 2,41 & 7,55 & 5,48 & 13,0 & 100 & 100 & 100 & 100 & 100 \\
\hline \multicolumn{16}{|l|}{ Produtos } \\
\hline$d>2,2$ & 94,2 & 69,9 & 8,18 & 3,36 & 1,12 & 1,44 & 2,48 & 6,58 & 4,82 & 11,4 & 96,3 & 94,8 & 95,0 & 82,5 & 97,5 \\
\hline$d>2,5$ & 74,6 & 72,7 & 8,47 & 3,56 & 0,99 & 1,67 & 2,54 & 4,61 & 3,36 & 7,97 & 79,3 & 77,7 & 79,7 & 45,6 & 82,8 \\
\hline$d>2,6$ & 53,9 & 73,5 & 7,93 & 3,98 & 1,13 & 1,80 & 1,81 & 4,65 & 3,21 & 7,86 & 58,0 & 52,5 & 64,3 & 32,5 & 51,2 \\
\hline$d>2,64$ & 25,2 & 60,3 & 11,0 & 5,97 & 2,37 & 2,41 & 2,14 & 7,87 & 5,27 & 13,1 & 22,2 & 34,2 & 45,2 & 25,4 & 31,7 \\
\hline
\end{tabular}

A distribuição em massa acumulada nos produtos afundados mostra um comportamento similar para as distintas frações granulométricas, indicando que o material já se encontra com boa liberação na malha de cominuição adotada. No entanto, verifica-se ainda uma tendência de aumento na proporção em massa de materiais acima de $2,5 \mathrm{~g} / \mathrm{cm} 3$ para as frações mais finas, que pode ocorrer por liberação entre as fases, ou variação na composição das frações (a composição é detalhada nos resultados do MLA).

Quase a totalidade de massa da amostra encontra-se acima de 2,2 $\mathrm{g} / \mathrm{cm} 3$, sendo os materiais mais leves compostos por materiais orgânicos. As curvas de liberação indicam que uma 
parcela expressiva de material (de 70 a 80\%) encontra-se nos produtos afundados em 2,5 g/cm3, os quais concentram partículas de menor porosidade. Comportamento distinto é verificado para os produtos afundados em $2,64 \mathrm{~g} / \mathrm{cm} 3$ (até $40 \%$ em massa), onde maior proporção de material é observada nas frações mais grossas, pela concentração de fragmentos líticos resultantes da britagem das rochas.

A composição química determinada por FRX confirma os resultados da análise mineralógica por DRX e as interpretações das observações ao microscópio estereoscópico. Os elevados teores de $\mathrm{CaO}+\mathrm{PF}$ nos produtos $\mathrm{d}<2,2$ (39,5\% para a amostra total) e 2,2<d<2,5 (24,5\%) estão relacionados à presença de materiais cimentícios. No produto $2,2<\mathrm{d}<2,5$ verifica-se o aumento nos teores de alumina e $\mathrm{K} 2 \mathrm{O}$, já o produto $2,60<\mathrm{d}<2,64$ é constituído essencialmente por quartzo $(85,1 \%)$ com baixos teores de $\mathrm{CaO}+\mathrm{PF}$, enquanto no produto mais pesado, ocorre um decréscimo no teor de quartzo e aumento na alumina, óxido de ferro, álcalis e também no conteúdo de cálcio e perda ao fogo.

As distribuições dos elementos indicam que apesar do produto $d<2,2$ representar apenas $5,8 \%$ da massa, este é responsável por $17,5 \%$ do total contido de $\mathrm{CaO}+\mathrm{PF}$; se considerados os produtos abaixo de $2,5 \mathrm{~g} / \mathrm{cm} 3$ (25,4\% da massa) este valor ultrapassa os $50 \%$.

Considerando-se os totais acumulados nos produtos afundados, observa-se que a remoção do flutuado em 2,5 gera um produto com $74,6 \%$ da massa e apenas $45,6 \%$ de CaO+PF e cerca de $80 \%$ de sílica, alumina, ferro, $\mathrm{Na2O}+\mathrm{K} 2 \mathrm{O}$. Considerando-se apenas os produtos afundados em 2,6 , tem-se uma recuperação de $53,9 \%$ da massa, $32,5 \%$ de $\mathrm{CaO}+\mathrm{PF}, 58,0 \%$ de sílica e $51,2 \%$ de $\mathrm{Na} 2 \mathrm{O}+\mathrm{K} 2 \mathrm{O}$.

A soma dos teores de $\mathrm{CaO}+\mathrm{PF}$, para os produtos obtidos na separação densitária, torna evidente a concentração preferencial destes nos produtos de menor densidade, confirmando a correlação com o conteúdo de pasta de cimento porosa. A diminuição nos teores desses elementos, com o aumento da densidade, corroboram essa informação.

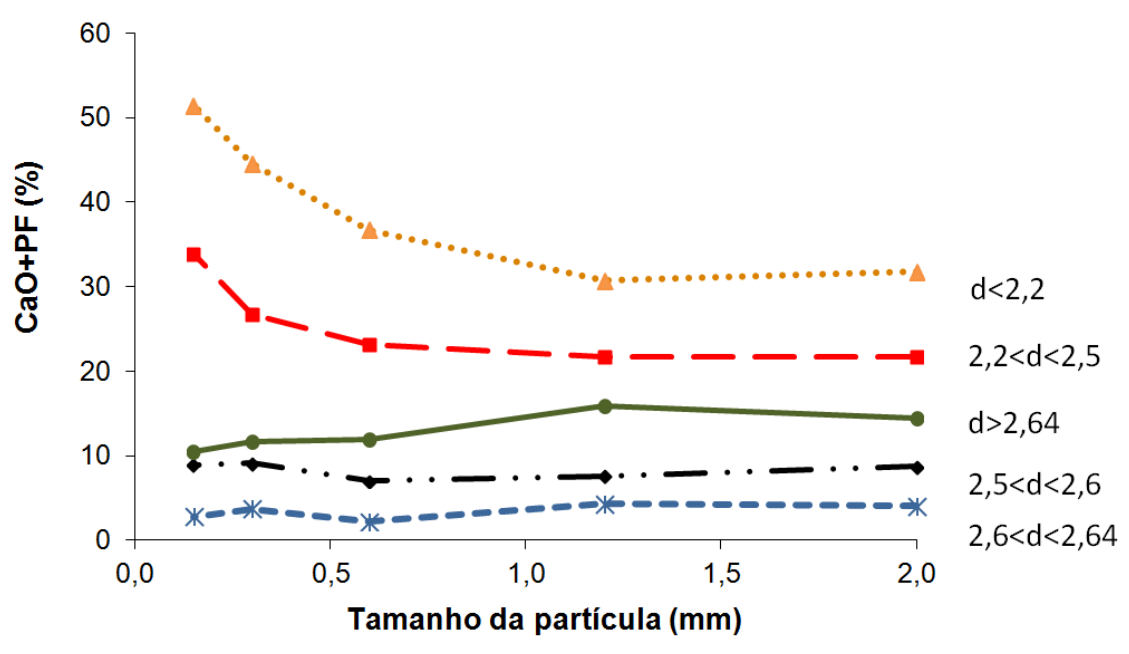

Figura 2 - Teor de CaO+PF nos produtos da separação por densidade

No entanto, para o produto afundado em $2,64 \mathrm{~g} / \mathrm{cm} 3$, ocorre um acréscimo na soma desses teores. Considerando que acima dessa densidade têm-se essencialmente minerais de maiores densidade, pode-se inferir que o aumento do conteúdo de $\mathrm{CaO}+\mathrm{PF}$ não esteja relacionado à pasta 
de cimento e sim a outras fases portadoras de cálcio. Essa constatação motivou o desenvolvimento de uma rotina de análise de imagens para identificação dessas fases, não passíveis de quantificação por análises químicas.

\subsection{Análises quantitativas das fases por MEV-MLA}

A composição dos produtos, em termos de fases constituintes, é apresentada na Tabela 2, sendo os totais, por produtos de densidade, mostrados na Figura 2. As principais fases presentes identificadas na amostra são quartzo (53\% em média), feldspatos (23\%) e a pasta de cimento (10\%); fases secundárias são representadas por carbonatos (calcita+dolomita, totalizando 3,5\%), cerâmica $(3,4 \%)$, micas $(3,5 \%)$, piroxênio+anfibólio $(2,2 \%)$. Outros minerais menores $(1,1 \%)$ são representados por apatita, hematita, ilmenita, titanita e zircão.

Nas frações acima de 1,2 mm o conteúdo de pasta no produto $\mathrm{d}<2,2$ é da ordem de 30 a $40 \%$ e os conteúdos de quartzo e feldspatos somam 50 a $60 \%$; nas frações mais finas verifica-se uma crescimento progressivo nos teores de pasta de cimento no produto de menor densidade, de $51 \%, 71 \%$ e $81 \%$, indicando maior liberação entre a pasta de cimento es demais fases. A fração mais fina $(-0,3+0,15 \mathrm{~mm})$ apresenta um comportamento ligeiramente diferente devido ao aumento de pasta de cimento. Nas demais frações (abaixo de 1,2 e acima de 0,15 mm), o conteúdo de quartzo aumenta de 35 a 64\% para as frações mais finas; o inverso ocorre para os feldspatos com teores decrescentes de 36 para 16\%, demonstrando concentração preferencial destes nas frações mais grossas, juntamente com os minerais máficos.

A pasta de cimento concentra-se essencialmente no produto flutuado em $2,2 \mathrm{~g} / \mathrm{cm} 3 \mathrm{e}$, secundariamente, no produto entre 2,2 e $2,5 \mathrm{~g} / \mathrm{cm} 3$. Os feldspatos estão presentes em todos os produtos acima de $2,2 \mathrm{~g} / \mathrm{cm} 3$, mas concentram-se preferencialmente nos produtos $2,5<\mathrm{d}<2,6 \mathrm{e}$ $\mathrm{d}>2,64 \mathrm{~g} / \mathrm{cm} 3$. Analogamente, o quartzo destaca-se no produto $2,6<\mathrm{d}<2,64 \mathrm{~g} / \mathrm{cm} 3$, onde representa cerca de $80 \%$ em massa do produto.

Tabela 2 - Composição dos produtos das separações em líquido denso determinada por MEV-MLA, total calculado $-3,0+0,15 \mathrm{~mm}$

\begin{tabular}{|c|c|c|c|c|c|c|c|c|c|c|c|c|c|c|c|c|c|c|c|c|c|c|c|c|c|}
\hline \multirow{2}{*}{ Produto } & \multicolumn{5}{|c|}{$-3,0+2,0 \mathrm{~mm}$} & \multicolumn{5}{|c|}{$-2,0+1,2 \mathrm{~mm}$} & \multicolumn{5}{|c|}{$-1,2+0,6 \mathrm{~mm}$} & \multicolumn{5}{|c|}{$-0,6+0,3 \mathrm{~mm}$} & \multicolumn{5}{|c|}{$-0,3+0,15 \mathrm{~mm}$} \\
\hline & 17 & $\mathrm{fd}$ & pc & ca & ou & $q z$ & $\mathrm{fd}$ & pc & ca & $u$ & $q z$ & $\mathrm{fd}$ & $\mathrm{pc}$ & ca & 0 & $q z$ & $\mathrm{fd}$ & $\mathrm{pc}$ & $\mathrm{ca}$ & 1 & $q z$ & $\mathrm{fd}$ & pc & ca & H \\
\hline & & 5,7 & 30 & 2,7 & 5,4 & 6 & 6,1 & 39 & 3,3 & 5,1 & 6 & 5,5 & 51 & 2,5 & 4,9 & 6 & 5,6 & 71 & 1,8 & 5,0 & 9,4 & 3,9 & 81 & 2,3 & 3,7 \\
\hline & 52 & 21 & 16 & 4,8 & 5,5 & 57 & 17 & 18 & 2,9 & 5,4 & 47 & 19 & 22 & 2,5 & 8,9 & 48 & 17 & 27 & 2,3 & 6,4 & & 14 & 49 & 3,6 & 7,9 \\
\hline 6 & 27 & 43 & 8,1 & 4,3 & 17 & 39 & 43 & 4,5 & 1,0 & 12 & 54 & 35 & 4,7 & 0,8 & 4,9 & 49 & 37 & 7,7 & 1,0 & 5,3 & 59 & 26 & 9,6 & 1,1 & 4,0 \\
\hline & 43 & 42 & 1,9 & 3,1 & 9,7 & 62 & 31 & 1,6 & 0,2 & 6 & 79 & 17 & 1,2 & 0,3 & 2,2 & 88 & 7,6 & 1,8 & 0,2 & 2,2 & 87 & 8,0 & 2,2 & 0,5 & 2,4 \\
\hline$d>2,64$ & 23 & 44 & 1,2 & 7,8 & 24 & 32 & 34 & 1,7 & 11 & 22 & 43 & 25 & 1,4 & 8,7 & 22 & 40 & 17 & 1,8 & 9,7 & 26 & 51 & 11 & 2,1 & 8,1 & 27 \\
\hline otal & 35 & 36 & 7,8 & 5,6 & 16 & 47 & 29 & 8,7 & 4,3 & 12 & 56 & 22 & 9,1 & 3,1 & 9,1 & 64 & 16 & 10 & 2,4 & 7,7 & 60 & 14 & 15 & 2,5 & 8,1 \\
\hline
\end{tabular}

Note: qz - quartzo, fd - feldspato, pc - pasta de cimento, ca - carbonatos, ou - outros (mica+piroxênio+ anfibólio+outros) 


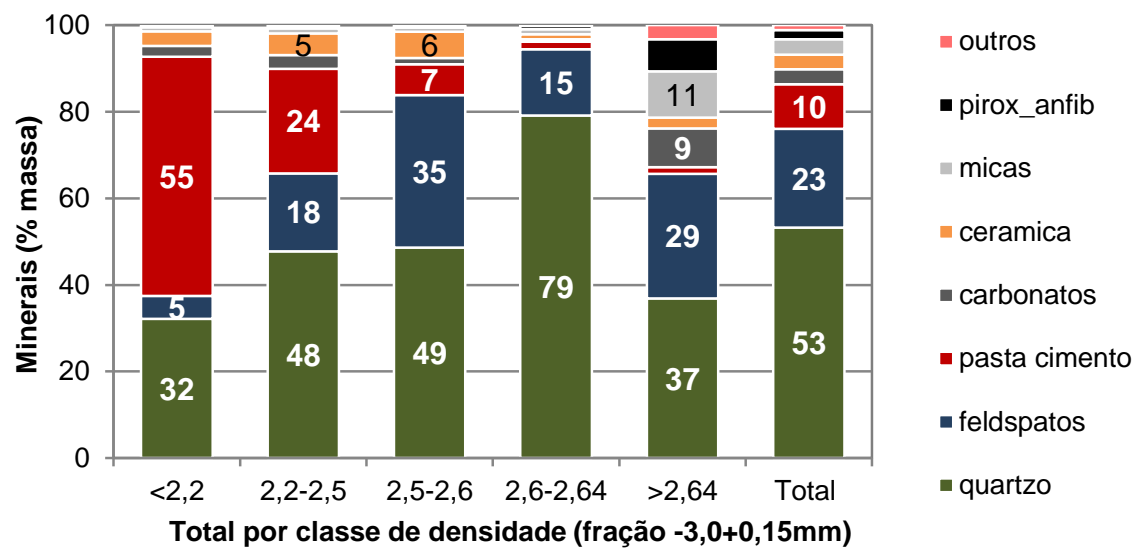

Figura 2 - Distribuição das fases nos produtos da separação por densidade

$\mathrm{O}$ aumento dos teores de $\mathrm{CaO}+\mathrm{PF}$ no produto $\mathrm{d}>2,64 \mathrm{~g} / \mathrm{cm} 3$ observados nos resultados de análises químicas (Tabela 1, Figura 2) deve-se à presença de carbonatos nesse produto (9\%), não observados nos produtos mais leves. Adicionalmente, nota-se também um aumento expressivo na proporção de mica, piroxênios e anfibólios.

Considerando-se as distintas frações granulométricas, o conteúdo de quartzo aumenta nas frações mais finas, ao passo os teores de feldspato e carbonatos diminuem.

Os teores de pasta de cimento e das princiais fases minerais da amostra (quartzo+feldspato+carbonatos+piroxênio/anfibólio) variam essencialmente com a densidade da separação, tal como exposto na Figura 3, confirmando os resultados das análises químicas. A partir da identificação ao MLA, é possível a diferenciação das fases portadoras de cálcio, que apresentam comportamento completamente inverso, ou seja, a pasta de cimento concenta-se nas densidades menores enquanto os carbonatos preferencialmente nos produtos acima de $2,64 \mathrm{~g} / \mathrm{cm} 3$.
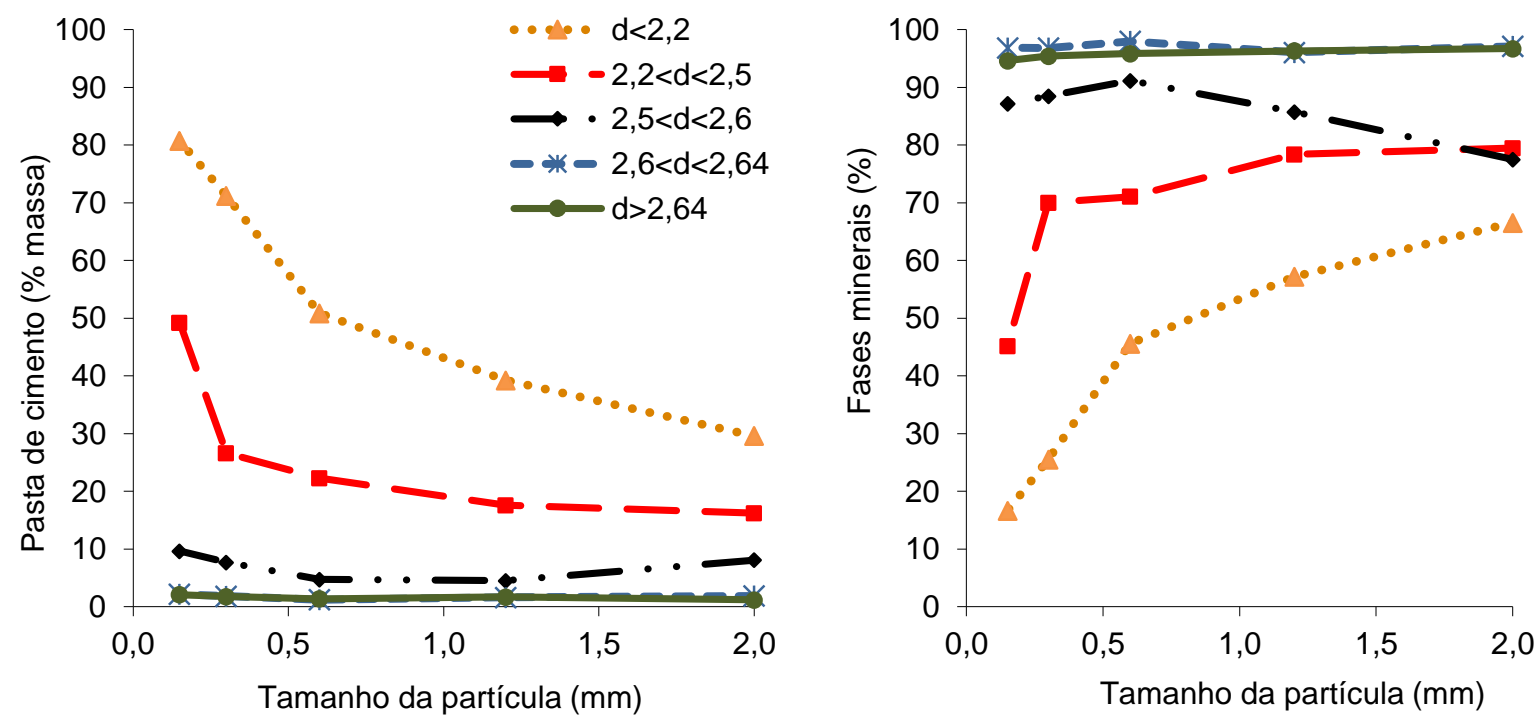

Figura 3 - Distribuição das fases nos produtos da separação por densidade 


\section{CONCLUSÕES}

A caracterização das associações dos agregados miúdos reciclados separados por líquido denso em diferentes densidades confirmou a separabilidade entre as fases enriquecidas em pasta de cimento e as demais fases minerais provenientes da cominuição das rochas, suportando a realização de estudos de beneficiamento neste produto.

Ressalta-se que o MLA permite o entendimento do comportamento dos agregados miúdos reciclados quando submetidos a processos de remoção da pasta de cimento ou a operações unitárias de tratamento de minérios para separação das fases porosas ricas em pasta cimentícia. No entanto, trata-se de uma ferramenta de elevada complexidade, que exige grande capacitação técnica na sua operação, relativamente morosa, com elevado custo, fatores que somados tornam este procedimento limitado (poucos sistemas disponíveis no país).

A estimativa do conteúdo de pasta de cimento a partir da soma dos teores de CaO+PF é precisa quando da ausência de agregados carbonáticos (calcita/dolomita), no entanto nos produtos de maior densidade e, portanto, de menor teor de pasta cimentícia, tal estimativa passa a ser ainda bastante equivocada, subestimando a qualidade de agregados miúdos reciclados de melhor qualidade.

\section{AGRADECIMENTOS}

Os autores agradecem a equipe do Centro de Tecnologia de Processos da Metso, e a Urbem Tecnologia Ambiental.

\section{REFERÊNCIAS BIBLIOGRÁFICAS}

1. ANGULO, S. C. Desenvolvimento de novos mercados para a reciclagem massiva de RCD. In: Seminário de Desenvolvimento Sustentável e a Reciclagem na Construção Civil, 2002a. São Paulo. Proceedings.São Paulo: IBRACON/IPEN, 2002a. p. 293-307.

1. ANGULO, S. C. Caracterização de agregados de resíduos de construção e demolição reciclados e a influência de suas características no comportamento dos concretos. 2005. 149 p. Tese (Doutorado) - Escola Politécnica, Universidade de São Paulo, São Paulo.

2. ANGULO, S. C.; ULSEN, C.; JOHN, V. M.; KAHN, H.; CINCOTTO, M. A. Chemical-mineralogical characterization of C\&D waste recycled aggregates from Sao Paulo, Brazil. Waste Management, v. 29, n. 2, p. 721-730, 2009.

3. BARRA, M.; VAZQUEZ, E. Properties of concretes with recycled aggregates: influence of properties of the aggregatesand their interpretation. In: Sustainable construction: use of recycled concrete aggregate, 1998. London. Proceedings. London: 1998. p. 19-30.

4. DE JUAN, M. S.; GUTIERREZ, P. A. Study on the influence of attached mortar content on the properties of recycled concrete aggregate. Construction and Building Materials, v. 23, n. 2, p. 872-877, 2009.

5. FANDRICH, R.; GU, Y.; BURROWS, D.; MOELLER, K. Modern SEM-based mineral liberation analysis. International Journal of Mineral Processing, v. 84, p. 310-320, 2007.

6. HENDRIKS, C. F. A new vision on the building cycle. Holanda: Aeneas, 2004. 251 p. 
7. ULSEN, C. Caracterização tecnológica de resíduos de construção e demolição. 2006. 171 p. Tese (Mestrado) - Escola Politécnica, Universidade de São Paulo, São Paulo.

8. ULSEN, C. Caracterização e separabilidade de agregados miúdos produzidos a partir de resíduos de construção e demolição. 2011. 222 p. Tese (Doutorado) - Departamento de Engenharia de Minas e de Petróleo, Universidade de São paulo, São Paulo.

9. ULSEN, C.; HAWLITSCHEK, G.; KAHN, H.; ANGULO, S. C.; JOHN, V. M. Technological Characterization of Fine Fraction from brazilian C\&D Waste. In: JOHN, V. M.;VAZQUEZ, E., et al, 2nd International RILEM Conference on Progress of Recycling in the Built Environment, 2010. São Paulo. Proceedings.São Paulo: RILEM Publications SARL, 2010. p. 449-458.

10. ULSEN, C.; KAHN, H.; HAWLITSCHEK, G.; MASINI, E. A.; ANGULO, S. C.; JOHN, V. M. Production of recycled sand from construction and demolition waste. Construction and Building Materials, v. 40, n. 0, p. 1168-1173, 2013. 